Supporting Information

\title{
Cellular Nanofiber Structure with Secretory Activity-Promoting Characteristics for Multicellular Spheroid Formation and Hair Follicle Regeneration
}

Kexin Zhang ${ }^{a}$, Xiufeng Bai ${ }^{b}$, Zhipeng Yuan ${ }^{a}$, Xintao Cao ${ }^{b c}$, Xiangyu Jiao ${ }^{a}$, Yan Qin $^{b *}$, Yongqiang Wen ${ }^{a *}$, Xueji Zhang ${ }^{a}$

${ }^{a}$ Research Center for Bioengineering and Sensing Technology, School of Chemistry and Biological Engineering, University of Science and Technology Beijing, Beijing 100083, P. R. China

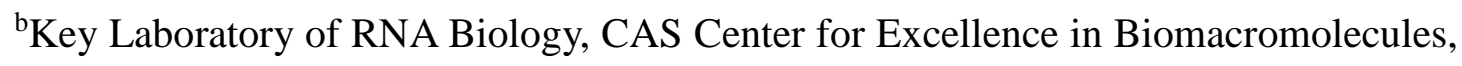
Institute of Biophysics, Chinese Academy of Sciences, 15 Datun Road, Chaoyang District, Beijing 100101, China

${ }^{c}$ University of Chinese Academy of Sciences, Beijing 100049, China

Corresponding Author:

Yongqiang Wen

E-mail: wyq_wen@ustb.edu.cn; Tel: +86 82375840

Yan Qin

E-mail: qiny@ibp.ac.cn; Tel:_+8664869250 


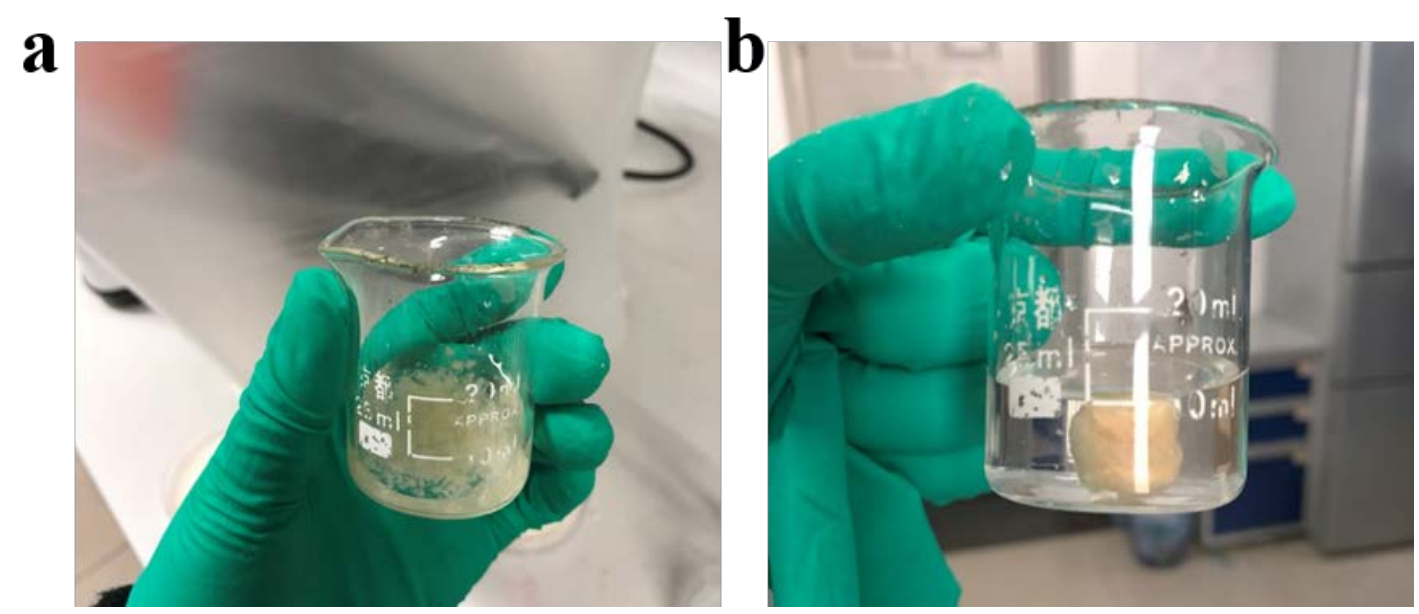

Fig. S1. Morphological changes in water before (a) and after (b) heat treatment.

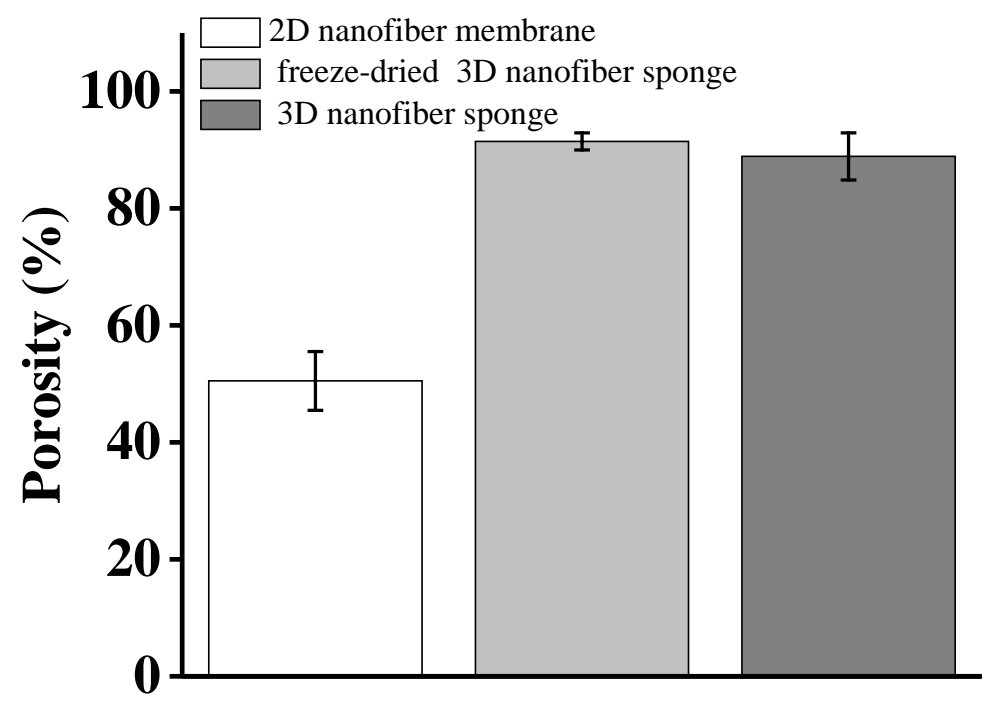

Fig. S2. Porosities of the 2D nanofiber membrane, freeze-dried 3D nanofiber sponge and 3D nanofiber sponges (after heat treatment). The porosity $(\mathrm{P})$ is calculated using the following equation: $\mathrm{P}=\left[\left(\mathrm{V}-\mathrm{V}_{0}\right) / \mathrm{V}\right] \times 100 \%, \mathrm{~V}=\mathrm{L}$ (length) $\times \mathrm{W}$ (width) $\times \mathrm{T}$ (thickness) [where $\mathrm{V}$ is the volume of the nanofiber], and $\mathrm{V}_{0}=\left(\left(\mathrm{m}_{0}\right) /\left(\rho_{0}\right)\right)$ [where $\mathrm{m}_{0}$ is the mass of the bulk CS materials and bulk PVA materials, and $\rho_{0}$ is the density of bulk CS materials and bulk PVA materials]. 


\section{Morphology and immunofluorescent staining of DPCs.}

DPCs were integrally obtained by combining microdissection and enzymatic digestion methods. After the DPs were cultured on the culture dish, the DPCs migrated out of the DPs on day 1. The DPs began to disintegrate and more DPCs grow from DPs on day 3. As the cells proliferated, a cell morphology of confluent flat was observed on day 7 (Fig.S3a). DPCs were positive for the expression of dermal papilla marker $\alpha$ SMA and vimentin, and negative for the expression of epidermal marker Krt 14(Fig. S3b). These results indicated that we have obtained DPCs successfully.

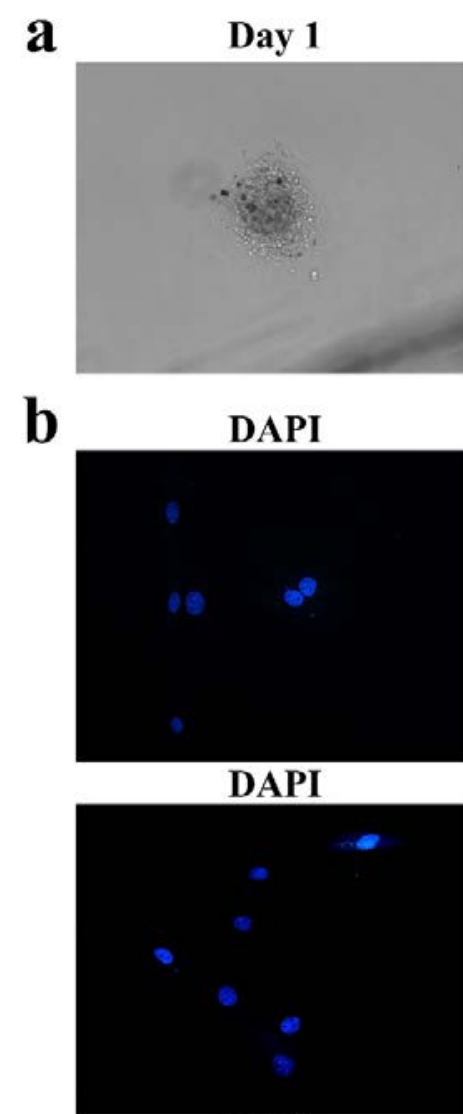

DAPI

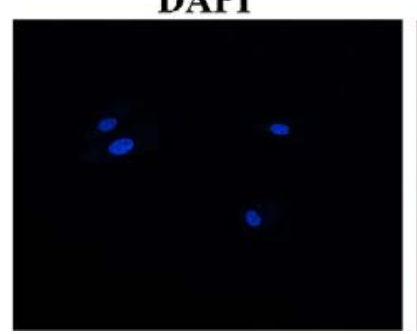

Day 3

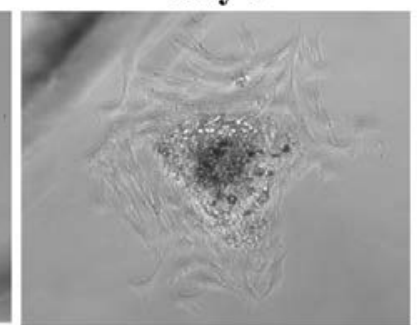

a-SMA

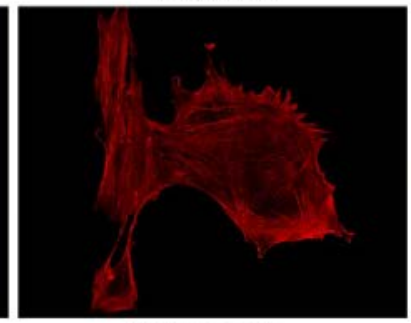

Vimentin

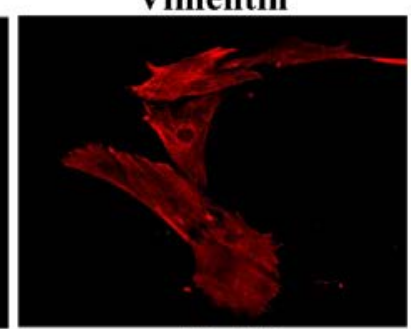

Krt14

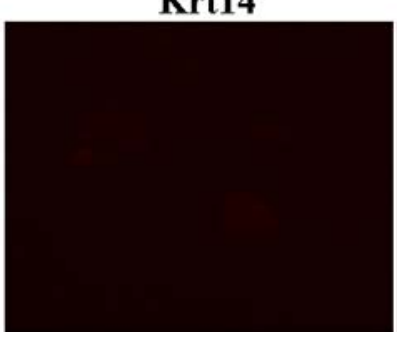

Day 7

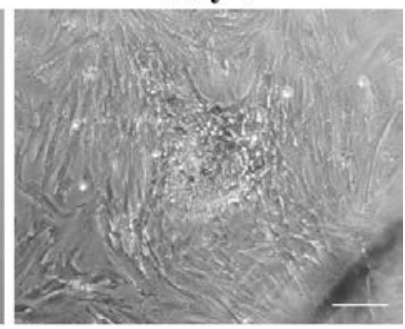

Merge

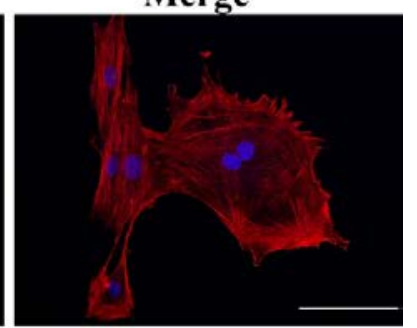

Merge

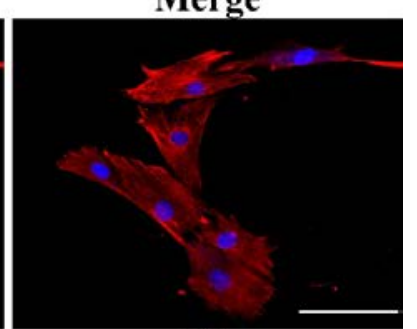

Merge

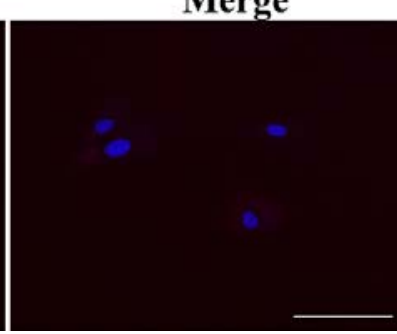

Fig. S3. The preparation of DPCs. (a)The morphological changes of DP at different time points (days). scale bar $=200 \mu \mathrm{m}$. (b) Immunofluorescence staining of DPCs. scale bar $=50 \mu \mathrm{m}$ 


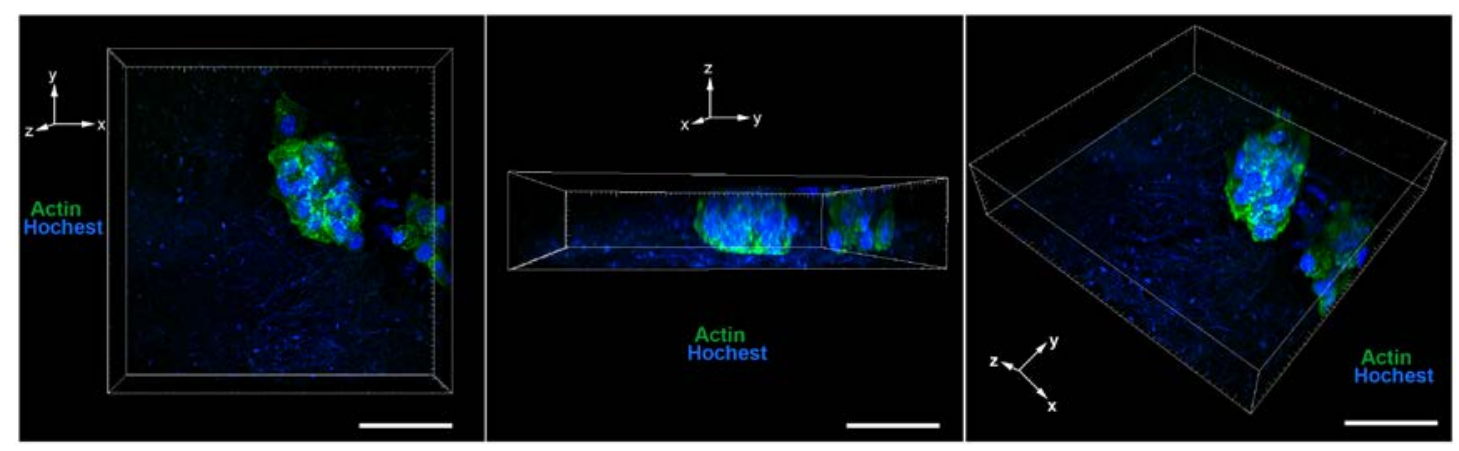

Fig. S4. The 3D reconstruction of DP microtissue cultured on 3D cellular structure nanofiber sponge (the cell densitiy was $1 \times 10^{5} \mathrm{cell} / \mathrm{cm}^{2}$ ). The cell actin and nuclei were stained with Actin-Tracker (green) and Hoechst (blue), respectively; scale bar $=35 \mu \mathrm{m}$.

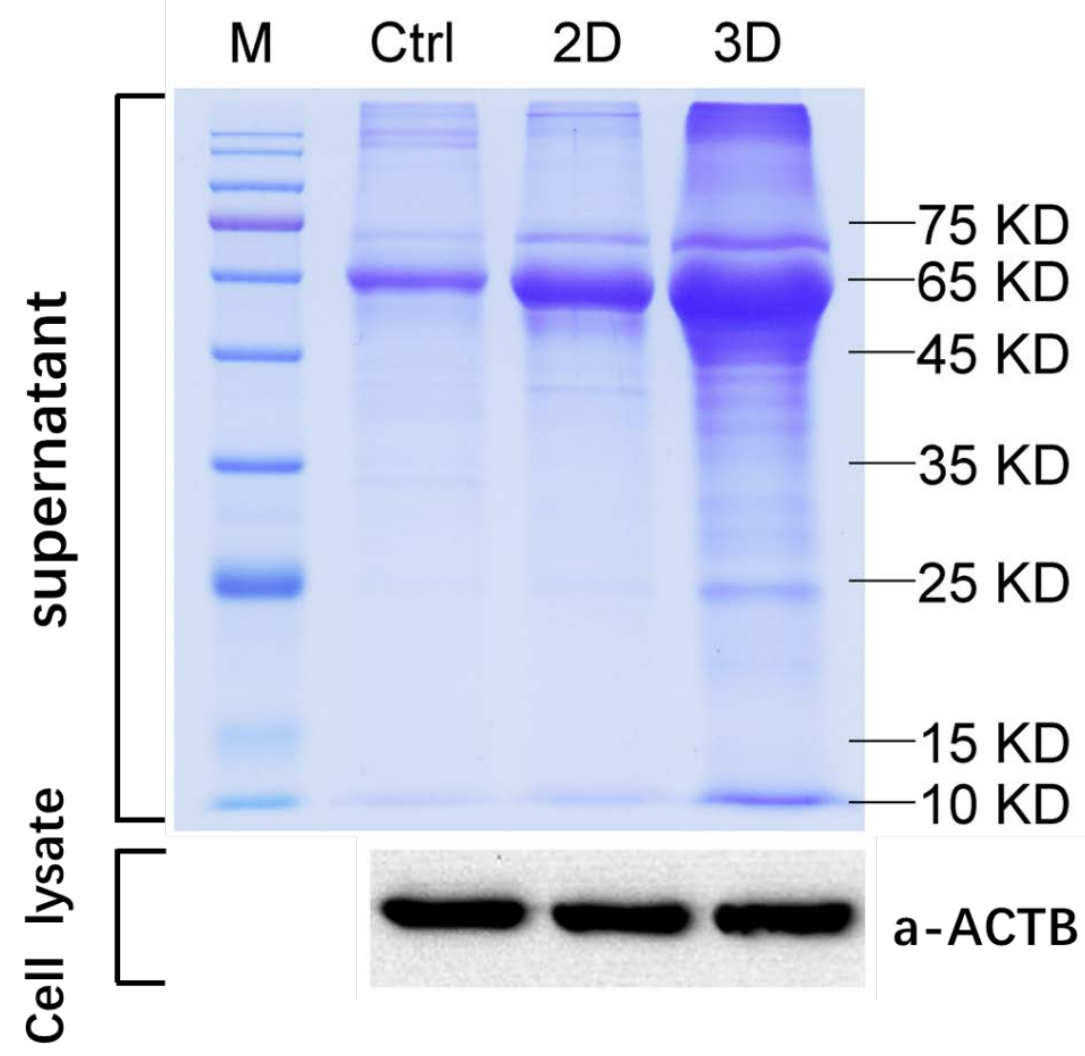

Fig. S5. The secreted protein abundance was tested using electrophoresis. And the secreted protein was secreted by same amount of DPCs in control group, 2D group and 3D group. 


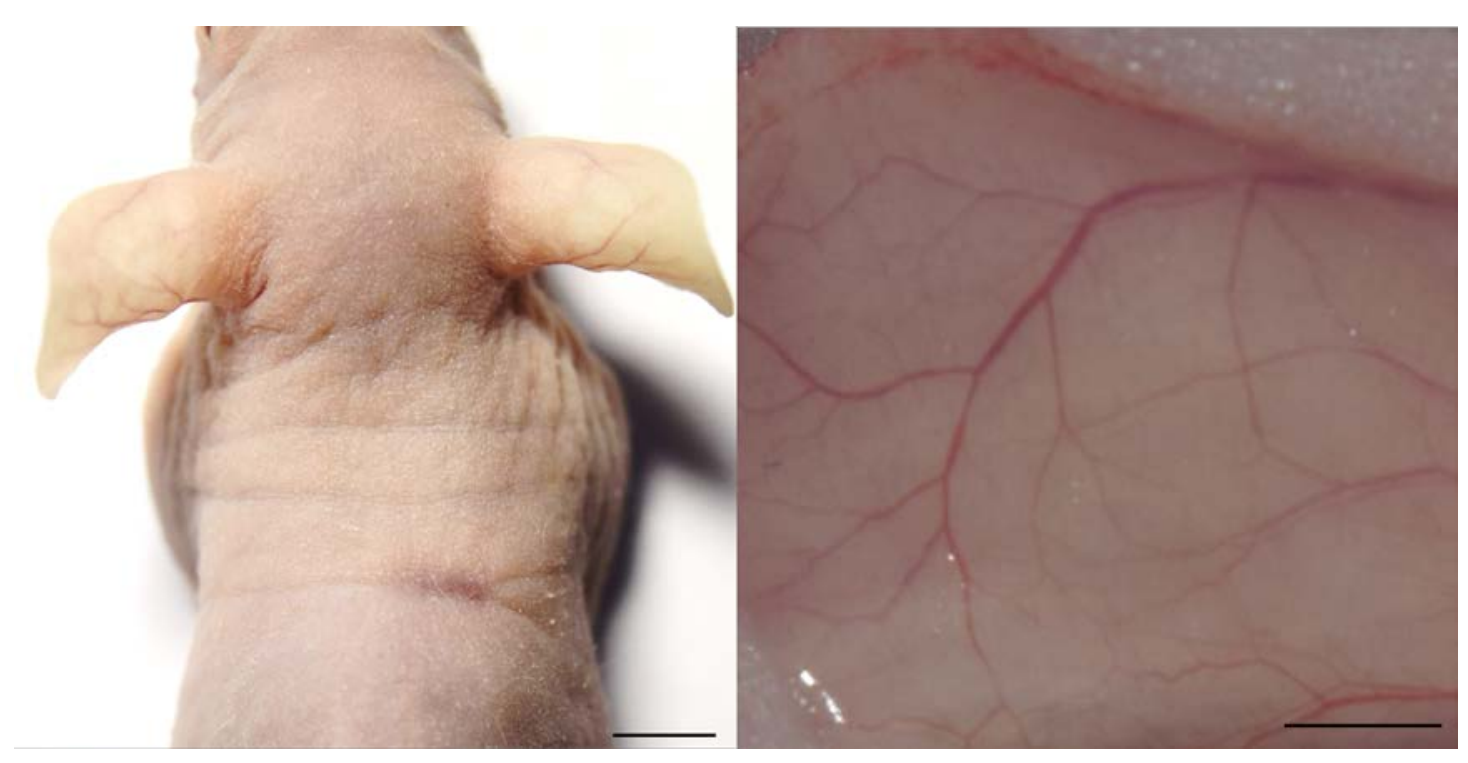

Fig. S6. In chamber (left) and patch (right) assay, the photos showed that transplanting epidermal cells alone cannot regenerated hair follicles after 4 weeks. (scale bar $=5 \mathrm{~mm}$ (left) and $1 \mathrm{~mm}$ (right))

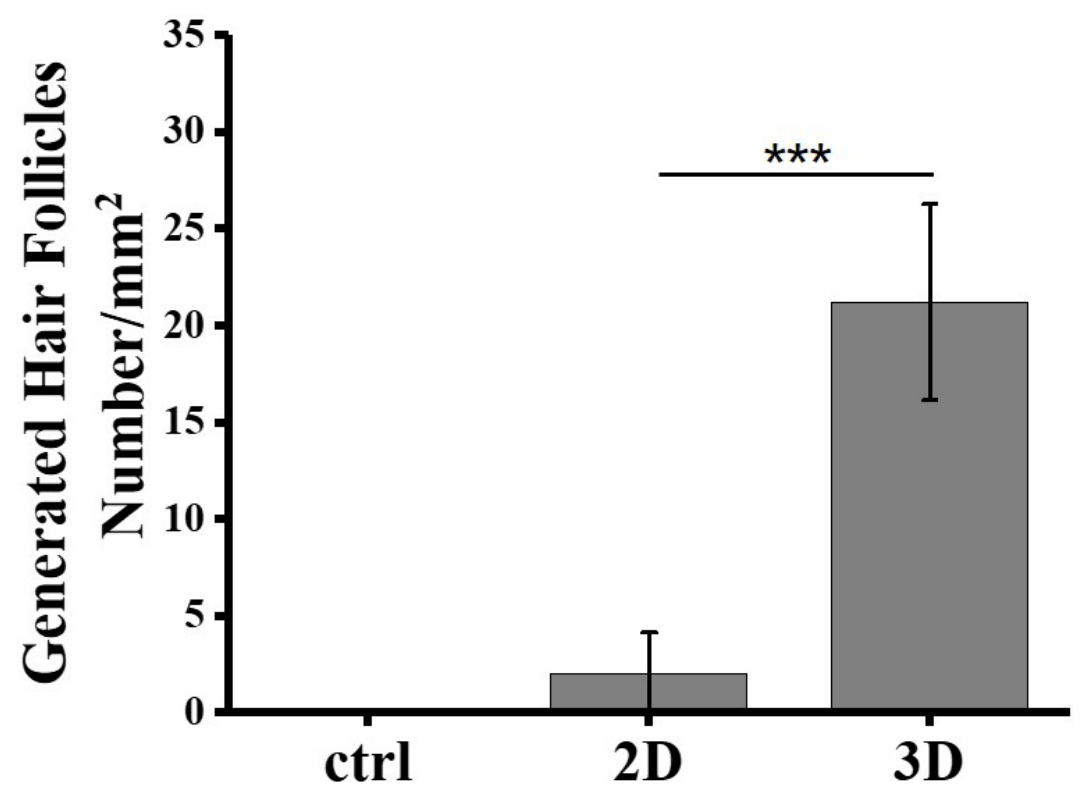

Fig. S7. In chamber assay, the statistics of generated hair follicles in control group, 2D group and $3 \mathrm{D}$ group $\left(* \mathrm{p}<0.05\right.$, ** $\mathrm{p}<0.01$, $\left.{ }^{* * *} \mathrm{p}<0.001\right)$. 